\title{
Efimov effect in quantum magnets
}

\author{
Yusuke Nishida.* Yasuyuki Kato, and Cristian D. Batista \\ Theoretical Division, Los Alamos National Laboratory, Los Alamos, New Mexico 87545, USA
}

(Dated: August 2012)

\begin{abstract}
Physics is said to be universal when it emerges regardless of the underlying microscopic details. A prominent example is the Efimov effect, which predicts the emergence of an infinite tower of threebody bound states obeying discrete scale invariance when the particles interact resonantly. Because of its universality and peculiarity, the Efimov effect has been the subject of extensive research in chemical, atomic, nuclear and particle physics for decades. Here we employ an anisotropic Heisenberg model to show that collective excitations in quantum magnets (magnons) also exhibit the Efimov effect. We locate anisotropy-induced two-magnon resonances, compute binding energies of three magnons and find that they fit into the universal scaling law. We propose several approaches to experimentally realize the Efimov effect in quantum magnets, where the emergent Efimov states of magnons can be observed with commonly used spectroscopic measurements. Our study thus opens up new avenues for universal few-body physics in condensed matter systems.
\end{abstract}

PACS numbers: 75.10.Jm, 75.30.Ds, 03.65.Ge, 03.65.Nk

\section{Introduction}

Sometimes we observe that completely different systems exhibit the same physics. Such physics is said to be universal and its most famous example is the critical phenomena [1]. In the vicinity of second-order phase transitions where the correlation length diverges, microscopic details become unimportant and the critical phenomena are characterized by only a few ingredients; dimensionality, interaction range and symmetry of the order parameter. Accordingly, fluids and magnets exhibit the same critical exponents. The universality in critical phenomena has been one of the central themes in condensed matter physics.

Similarly, we can also observe universal physics in the vicinity of scattering resonances where the $s$-wave scattering length diverges. Here low-energy physics is characterized solely by the $s$-wave scattering length and does not depend on other microscopic details. One of the most prominent phenomena in such universal systems is the Efimov effect, which predicts the emergence of an infinite tower of three-body bound states obeying discrete scale invariance:

$$
\frac{E_{n+1}}{E_{n}} \rightarrow \lambda^{-2} \quad(n \rightarrow \infty)
$$

with the universal scale factor $\lambda=22.6944$ [2]. Because of its universality and peculiarity, the Efimov effect has been the subject of extensive research in chemical, atomic, nuclear and particle physics for decades after the first prediction in 1970 3. 4. In particular, the recent experimental realization with ultracold atoms has greatly stimulated this research area [5].

In spite of such active research, the Efimov effect has not attracted much attention in condensed matter physics so far. However, because the Efimov effect is universal, it should emerge also in condensed matter systems. In this article, we show that collective excita- tions in quantum magnets (magnons) exhibit the Efimov effect by tuning an easy-axis exchange or singleion anisotropy. We will locate anisotropy-induced twomagnon resonances, compute the binding energies of three magnons and find that they fit into the universal scaling law. We will also propose several approaches to experimentally realize the Efimov effect in quantum magnets, including frustrated cases. So far multi-magnon bound states have been observed with different experimental techniques, but mostly in quasi-one-dimensional compounds [6-23]. Although the Efimov effect emerges only in three dimensions [3, 4], the same spectroscopic measurements can be used to observe the emergent Efimov states of magnons. Our study thus opens up new avenues for universal few-body physics in condensed matter systems. Also, in addition to the Bose-Einstein condensation of magnons 24, the Efimov effect provides a novel connection between atomic and magnetic systems.

\section{Anisotropic Heisenberg model}

To demonstrate the Efimov effect in quantum magnets, we consider an anisotropic Heisenberg model on a simple cubic lattice:

$$
\begin{aligned}
H= & -\frac{1}{2} \sum_{\boldsymbol{r}} \sum_{\hat{\boldsymbol{e}}}\left(J S_{\boldsymbol{r}}^{+} S_{\boldsymbol{r}+\hat{\boldsymbol{e}}}^{-}+J_{z} S_{\boldsymbol{r}}^{z} S_{\boldsymbol{r}+\hat{\boldsymbol{e}}}^{z}\right) \\
& -D \sum_{\boldsymbol{r}}\left(S_{\boldsymbol{r}}^{z}\right)^{2}-B \sum_{\boldsymbol{r}} S_{\boldsymbol{r}}^{z}
\end{aligned}
$$

where $\sum_{\hat{\boldsymbol{e}}}$ is a sum over six unit vectors; $\sum_{\hat{\boldsymbol{e}}= \pm \hat{\boldsymbol{x}}, \pm \hat{\boldsymbol{y}}, \pm \hat{\boldsymbol{z}}}$. Two types of uniaxial anisotropies are introduced here: anisotropy in the exchange couplings $\left(J_{z} \neq J\right)$ and singleion anisotropy $(D \neq 0)$ which generally exist owing to the crystal field and spin-orbit interaction. Spin operators $S_{\boldsymbol{r}}^{ \pm} \equiv S_{\boldsymbol{r}}^{x} \pm i S_{\boldsymbol{r}}^{y}$ and $S_{\boldsymbol{r}}^{z}$ obey the usual commutation relations $\left[S_{\boldsymbol{r}}^{+}, S_{\boldsymbol{r}^{\prime}}^{-}\right]=2 S_{\boldsymbol{r}}^{z} \delta_{\boldsymbol{r}, \boldsymbol{r}^{\prime}},\left[S_{\boldsymbol{r}}^{z}, S_{\boldsymbol{r}^{\prime}}^{ \pm}\right]= \pm S_{\boldsymbol{r}}^{ \pm} \delta_{\boldsymbol{r}, \boldsymbol{r}^{\prime}}$ and 
the identity $\left(S_{r}^{+}\right)^{2 S+1}=\left(S_{r}^{-}\right)^{2 S+1}=0$ for a spin $S$ representation. $J, J_{z}>0$ corresponds to a ferromagnetic coupling and $J, J_{z}<0$ to an antiferromagnetic coupling. In the latter case, by rotating spins by $\pi$ along the $z$-axis $\left(S_{r}^{ \pm} \rightarrow-S_{r}^{ \pm}\right)$only for sites with odd-valued $x+y+z$, we can choose $J>0$, which is assumed from now on.

The ground state for a sufficiently large magnetic field $B<0$ is a fully polarized state with all spins pointing downwards; $S_{r}^{z}|0\rangle=-S|0\rangle$ and $S_{r}^{-}|0\rangle=0$. Because of the U(1) symmetry of the Hamiltonian (2) under rotation $S_{\boldsymbol{r}}^{ \pm} \rightarrow e^{ \pm i \theta} S_{\boldsymbol{r}}^{ \pm}$, the relative magnetization $N \equiv \sum_{\boldsymbol{r}}\left(S_{\boldsymbol{r}}^{z}+S\right)$ is a conserved quantity. Accordingly, we can consider an excited state with fixed $N$, which corresponds to a particle number of magnons. $N$-magnon excitations on this ground state are described by a wave function $\Psi\left(\boldsymbol{r}_{1}, \ldots, \boldsymbol{r}_{N}\right) \equiv\left\langle 0\left|\prod_{i=1}^{N} S_{\boldsymbol{r}_{i}}^{-}\right| \Psi\right\rangle$, which is symmetric under any exchange of coordinates (Bose statistics) and satisfies the Schrödinger equation:

$$
\begin{aligned}
E \Psi\left(\boldsymbol{r}_{1}, \ldots, \boldsymbol{r}_{N}\right)=\left\langle 0\left|\left[\prod_{i=1}^{N} S_{\boldsymbol{r}_{i}}^{-}, H\right]\right| \Psi\right\rangle \\
=\left[\sum_{i=1}^{N} \sum_{\hat{e}} S J\left(1-\nabla_{\hat{\boldsymbol{e}}}^{i}\right)+\sum_{i<j}^{N}\left\{\sum_{\hat{e}} J \delta_{\boldsymbol{r}_{i}, \boldsymbol{r}_{j}} \nabla_{\hat{\boldsymbol{e}}}^{i}\right.\right. \\
\left.\left.\quad-\sum_{\hat{e}} J_{z} \delta_{\boldsymbol{r}_{i}, \boldsymbol{r}_{j}+\hat{\boldsymbol{e}}}-2 D \delta_{\boldsymbol{r}_{i}, \boldsymbol{r}_{j}}\right\}\right] \Psi\left(\boldsymbol{r}_{1}, \ldots, \boldsymbol{r}_{N}\right) .
\end{aligned}
$$

Here $\nabla_{\hat{\boldsymbol{e}}}^{i}$ is an operator to displace $\boldsymbol{r}_{i}$ to $\boldsymbol{r}_{i}+\hat{\boldsymbol{e}}$ and a constant energy shift $-\left(6 S J-6 S J_{z}-2 S D+D+B\right) N$ is omitted in the right hand side.

The emergence of the Efimov effect in quantum magnets can be understood intuitively by using an exact mapping between spins and bosons, which is known as the Holstein-Primakoff transformation [25]. It is clear from the Schrödinger equation (3) that $J$ in the first term acts as a hopping amplitude and gives a single-magnon dispersion relation:

$$
E_{0}(\boldsymbol{k})=\sum_{\hat{\boldsymbol{e}}} S J[1-\cos (\boldsymbol{k} \cdot \hat{\boldsymbol{e}})] .
$$

The rest describe interactions between a pair of magnons where $J_{z}>J$ and $D>0$ act as nearest-neighbour and on-site attractions, respectively plings, we can induce a scattering resonance between two magnons. Once the two-magnon resonance is achieved, three magnons should exhibit the Efimov effect (1) because the Efimov effect is universal in the sense that it emerges regardless of microscopic details.

\footnotetext{
$\dagger$ Note that the effective $N$-magnon hardcore repulsion also exists for $N=2 S+1$ according to $\left(S_{r}^{+}\right)^{2 S+1}=0$. By setting $\boldsymbol{r}_{1}=\cdots=\boldsymbol{r}_{N}$ in equation $(3)$, we obtain $[E-3 N(N-1) J+$ $N(N-1) D] \Psi(\boldsymbol{r}, \ldots, \boldsymbol{r})=0$. Therefore, unless $E-3 N(N-$ 1) $J+N(N-1) D=0$, the constraint $\Psi(\boldsymbol{r}, \ldots, \boldsymbol{r})=0$ is automatically satisfied. In particular, $D$ plays no role for $S=1 / 2$ as $\Psi(\boldsymbol{r}, \boldsymbol{r})=0$.
}

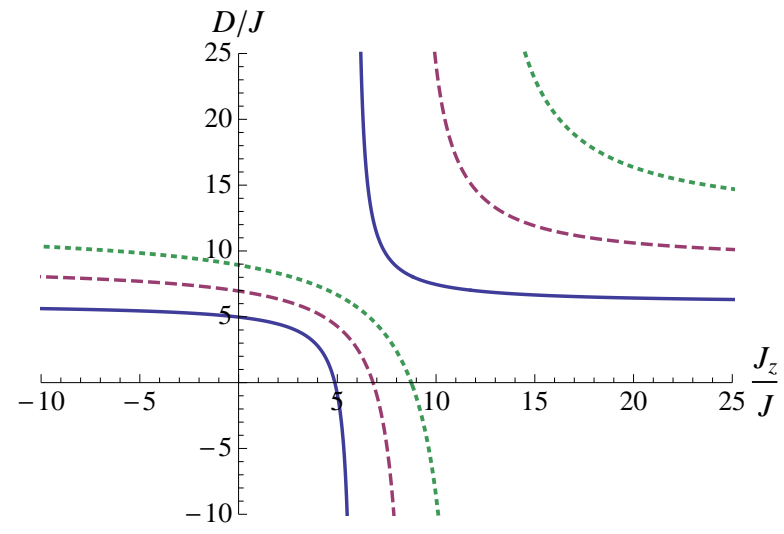

FIG. 1. Critical anisotropy for the two-magnon resonance. The solid, dashed and dotted curves are for $S=1$, $S=3 / 2$ and $S=2$, respectively. There exist(s) no, one or two $s$-wave bound state(s) at $\boldsymbol{K}=\mathbf{0}$ in the lower left, middle or upper right region, respectively, separated by the two critical curves. The appearance of two degenerate $d$-wave bound states is not indicated here.

\section{Two-magnon resonance}

We start with a scattering problem of two magnons. A two-magnon solution with a center-of-mass momentum $\boldsymbol{K}$ is written as

$$
\Psi\left(\boldsymbol{r}_{1}, \boldsymbol{r}_{2}\right)=e^{i \boldsymbol{K} \cdot \boldsymbol{R}} \psi_{\boldsymbol{K}}(\boldsymbol{\rho}),
$$

where $\boldsymbol{R} \equiv\left(\boldsymbol{r}_{1}+\boldsymbol{r}_{2}\right) / 2$ and $\boldsymbol{\rho} \equiv \boldsymbol{r}_{1}-\boldsymbol{r}_{2}$ are center-of-mass and relative coordinates, respectively. The Bose statistics of magnons implies $\psi_{\boldsymbol{K}}(\boldsymbol{\rho})=\psi_{\boldsymbol{K}}(-\boldsymbol{\rho})$ for the relative wave function. The two-magnon Schrödinger equation can be solved in a standard way by bringing it into the Lippmann-Schwinger equation (see Methods for details).

The scattering resonance between two magnons is defined by the divergence of the $s$-wave scattering length $\left(a_{s}\right)$ where a two-magnon bound state appears from the continuum. $a_{s}$ can be inferred from the asymptotic behaviour of the wave function at zero energy and zero center-of-mass momentum:

$$
\left.\lim _{|\boldsymbol{\rho}| \rightarrow \infty} \psi_{\mathbf{0}}(\boldsymbol{\rho})\right|_{E=0} \propto 1-\frac{a_{s}}{|\boldsymbol{\rho}|} .
$$

By matching this asymptotic behaviour with the obtained solution (equation (11) in Methods), the analytic expression of $a_{s}$ is obtained as

$$
\frac{a_{s}}{a}=\frac{\frac{3}{2 \pi}\left[1-\frac{D}{3 J}-\frac{J_{z}}{J}\left(1-\frac{D}{6 S J}\right)\right]}{2 S-1+\frac{J_{z}}{J}\left(1-\frac{D}{6 S J}\right)+3 W\left[1-\frac{D}{3 J}-\frac{J_{z}}{J}\left(1-\frac{D}{6 S J}\right)\right]} .
$$

Here $a$ is the lattice constant and $W \equiv$ $\frac{\sqrt{6}}{96 \pi^{3}} \Gamma\left(\frac{1}{24}\right) \Gamma\left(\frac{5}{24}\right) \Gamma\left(\frac{7}{24}\right) \Gamma\left(\frac{11}{24}\right) \quad=\quad 0.505462$ results from one of the Watson's triple integrals [26].

As a consequence, we find that the two-magnon resonance $a_{s} \rightarrow \infty$ takes place at $J_{z} / J=2.93654$ for 
TABLE I. Few lowest binding energies of three magnons right at the two-magnon resonances.

\begin{tabular}{|c|c|c|c|c|c|}
\hline$S$ & $J_{z} / J$ & $D / J$ & $n$ & $E_{n} / J$ & $\sqrt{E_{n-1} / E_{n}}$ \\
\hline \multirow[t]{3}{*}{$1 / 2$} & 2.93654 & - & 0 & $-2.09 \times 10^{-1}$ & - \\
\hline & & & 1 & $-4.15 \times 10^{-4}$ & 22.4 \\
\hline & & & 2 & $-8.08 \times 10^{-7}$ & 22.7 \\
\hline \multirow[t]{3}{*}{1} & 4.87307 & 0 & 0 & $-5.16 \times 10^{-1}$ & - \\
\hline & & & 1 & $-1.02 \times 10^{-3}$ & 22.4 \\
\hline & & & 2 & $-2.00 \times 10^{-6}$ & 22.7 \\
\hline \multirow[t]{2}{*}{1} & +1 & 4.76874 & 0 & $-5.50 \times 10^{-2}$ & - \\
\hline & & & 1 & $-1.16 \times 10^{-4}$ & 21.8 \\
\hline \multirow[t]{2}{*}{1} & -1 & 5.12703 & 0 & $-4.36 \times 10^{-3}$ & - \\
\hline & & & 1 & $-8.88 \times 10^{-6}$ & 22.2 \\
\hline- & - & - & $\infty$ & - & 22.6944 \\
\hline
\end{tabular}

$S=1 / 2$. Critical anisotropies for other spins are shown in Fig. 1. In particular, the two-magnon resonance for $S=1$ is induced by the exchange anisotropy at $J_{z} / J=4.87307(D=0)$, whereas it is induced by the single-ion anisotropy at $D / J=4.76874\left(J_{z}=J\right)$ or $D / J=5.12703\left(J_{z}=-J\right)$ for an isotropic ferromagnetic or antiferromagnetic coupling, respectively. Above these critical points, two magnons form an $s$-wave bound state at $\boldsymbol{K}=\mathbf{0}$. Its binding energy $E<0$ determines the three-magnon threshold in Figs. 2 and 3 below.

\section{Three-magnon Efimov effect}

We now turn to a bound-state problem of three magnons. A bound-state solution to the three-magnon Schrödinger equation can be obtained in a similar way to the previous two-magnon problem (see Methods for details). Because our purpose here is to demonstrate the Efimov effect of magnons, we focus on the $s$-wave channel at zero center-of-mass momentum where the Efimov effect is supposed to emerge.

We find that three magnons form a series of bound states and their binding energies are shown in Table I right at the anisotropy-induced two-magnon resonances located above. The ratios of two successive binding energies obey the universal scaling law (1), which supports that the observed bound states of three magnons are indeed the Efimov states. Note that although the Efimov effect emerges only in the $s$-wave channel [3, 4], it is in general possible that there are non-Efimov three-magnon bound states in other channels, which may have lower binding energies.

In the case of $S=1 / 2$, the two lowest binding energies near the two-magnon resonance are shown in Fig. 2. The universal theory predicts that the spectrum of Efimov states is completely characterized by the $s$-wave scatter- ing length $a_{s}$ and the so-called Efimov parameter $\kappa_{*}[4]$ :

$$
E_{n} \rightarrow-\lambda^{-2 n} \frac{\hbar^{2} \kappa_{*}^{2}}{m} F\left(\frac{\lambda^{n}}{\kappa_{*} a_{s}}\right) \quad(n \rightarrow \infty)
$$

Here $m$ is the mass of constituent particles and $F(x)$ is the universal function defined in a range $-0.663293 \leq$ $x \leq 14.1314$ and normalized as $F(0)=1$. The inverse effective mass of magnons is $1 / m=2 S J a^{2} / \hbar^{2}$, which is inferred from the single-magnon dispersion relation (4). By matching equation (8) with the binding energy of the second excited state at the resonance, we obtain $\kappa_{*} a \simeq$ 0.463 . The resulting universal curves for $n=0$ and $n=1$ are also plotted in Fig. 2 by using $a_{s}$ obtained in equation (7). We find an excellent agreement of the binding energy of the first excited state with the universal theory, which leaves no doubt that this three-magnon bound state is the universal Efimov state. On the other hand, the binding energy of the ground state deviates from the universal theory away from the two-magnon resonance where nonuniversal corrections become non-negligible.

Similarly, in the case of $S=1$ with $J_{z}=J(-J)$, we obtain $\kappa_{*} a \simeq 0.173(0.0478)$ by matching equation (8) with the binding energy of the first excited state at the resonance. The resulting universal curve for $n=0$ is plotted in Fig. 3 as well as the ground-state binding energy near the two-magnon resonance. We find a reasonable agreement between them, which confirms that this three-magnon bound state is consistent with the universal Efimov state. Our findings here support the fact that resonantly interacting magnons fall into the class of universal few-body systems. Accordingly, other universal aspects of Efimov physics, such as a pair of four-body resonances associated with every Efimov state [27, 28], also apply to the system of magnons. 

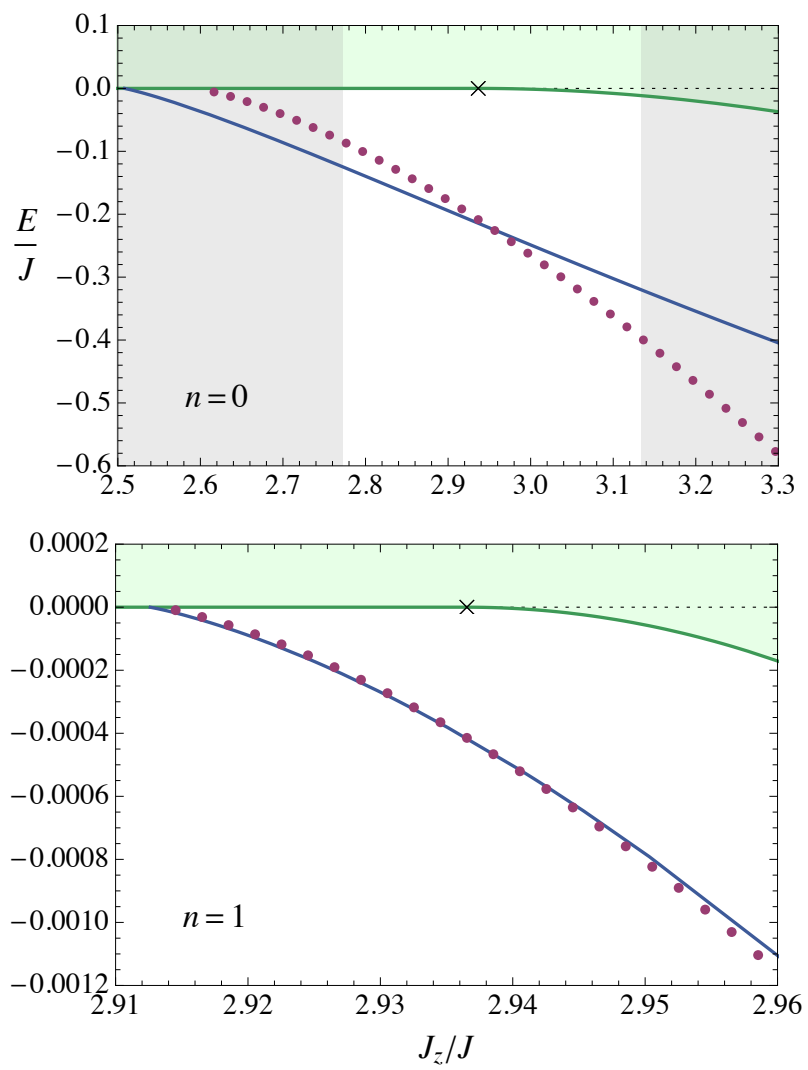

FIG. 2. Two lowest binding energies of three magnons for $S=1 / 2$ near the two-magnon resonance. Dots are numerical results and the solid curve is from the universal theory (8). The upper coloured region represents the threemagnon continuum and the cross $(x)$ marks the critical point where a two-magnon bound state appears. The left and right gray areas for $n=0$ indicate regions where $\left|a_{s}\right| / a<10$.

\section{Towards experimental realization}

In summary, we showed that magnons in quantum magnets exhibit the Efimov effect by tuning an easyaxis exchange or single-ion anisotropy. The single-ion anisotropy $D$ can be changed significantly in organic magnets by choosing different ligands [29]. Therefore, it is possible to find a compound with spin $S \geq 1$ whose $D / J$ ratio is already close to the critical value. For example, there is an $S=1$ ferromagnetic compound based on molecular $\mathrm{Ni}^{2+}$ squares with $D / J \simeq 3.0(5)$ [30], which is not far from the critical value 4.76874. Furthermore, the exchange coupling $J$ can be tuned with pressure 31 . to bring the system near the two-magnon resonance and realize the Efimov effect.

On the other hand, the single-ion anisotropy plays no role for $S=1 / 2$. In this case, the two-magnon resonance can be induced by the exchange anisotropy, although it is in general difficult to tune and its critical value $\left(J_{z} / J=2.93654\right)$ is somewhat large. However, the critical exchange anisotropy can be reduced
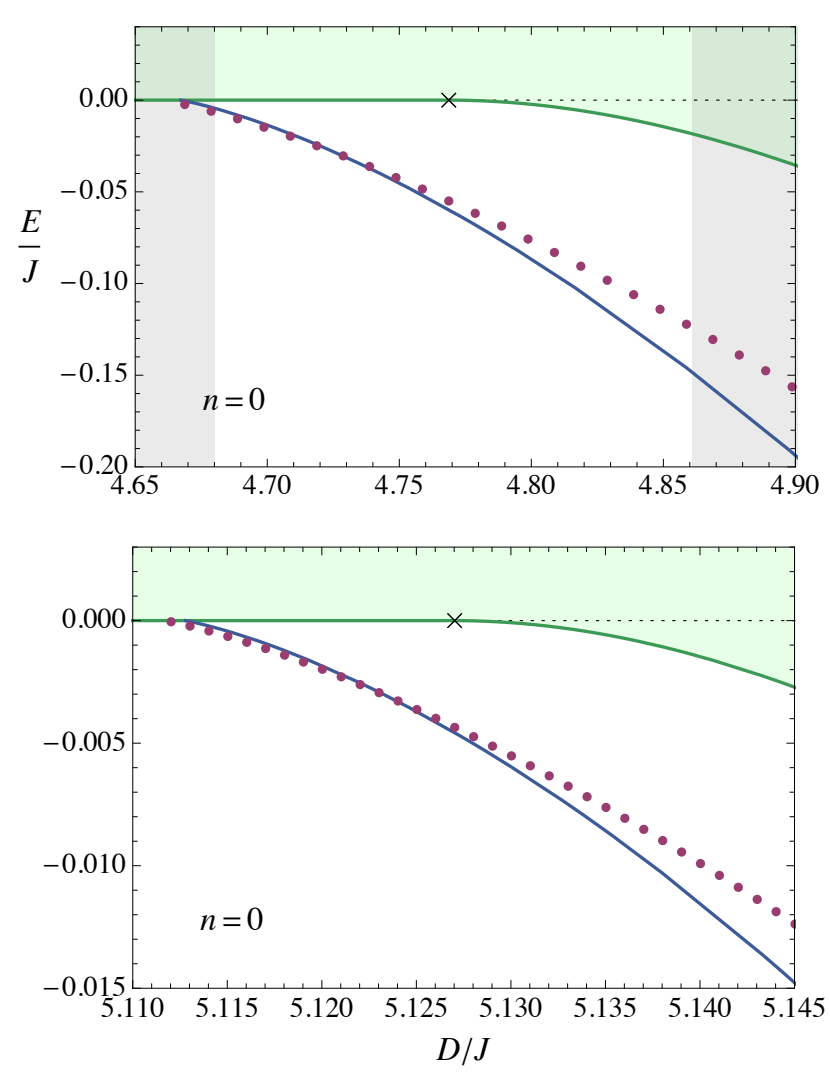

FIG. 3. Lowest binding energy of three magnons for $\boldsymbol{S}=1$ near the two-magnon resonance. The upper (lower) panel corresponds to $J_{z}=J(-J)$. See the legend of Fig. 2 for other details.

significantly if the magnet is spatially anisotropic. Spatially anisotropic exchange couplings can be taken into account simply by replacing $J$ and $J_{z}$ in the Hamiltonian $\left(22\right.$ with $J^{\hat{e}}$ and $J_{z}^{\hat{e}}$, respectively. We assume a uniaxial anisotropy $J \equiv J^{\hat{\boldsymbol{x}}}=J^{\hat{\boldsymbol{y}}}$ and $J_{z} \equiv J_{z}^{\hat{x}}=J_{z}^{\hat{y}}$ with a shared ratio $\gamma \equiv J / J^{\hat{z}}=J_{z} / J_{z}^{\hat{z}}$. As shown in Fig. 4, the corresponding critical exchange anisotropy reduces significantly towards an isotropic point $J_{z} / J \rightarrow 1$ in a quasi-one-dimensional $(\gamma \rightarrow 0)$ or two-dimensional $(\gamma \rightarrow \infty)$ limit. Because magnets with strong spatial anisotropies are very common 32 38, there is hope to find an $S=1 / 2$ ferromagnetic compound whose $J_{z} / J$ ratio is already close to the critical value. Once such a compound is identified, the spatial anisotropy $\gamma$ can be tuned with pressure.

An alternative approach to induce the two-magnon resonance in $S=1 / 2$ magnets even without the exchange anisotropy $\left(J_{z}=J\right)$ is to introduce frustrated exchange interactions. The simplest example is given by quasi-onedimensional spin chains with nearest-neighbour ferromagnetic and next-nearest-neighbour antiferromagnetic 


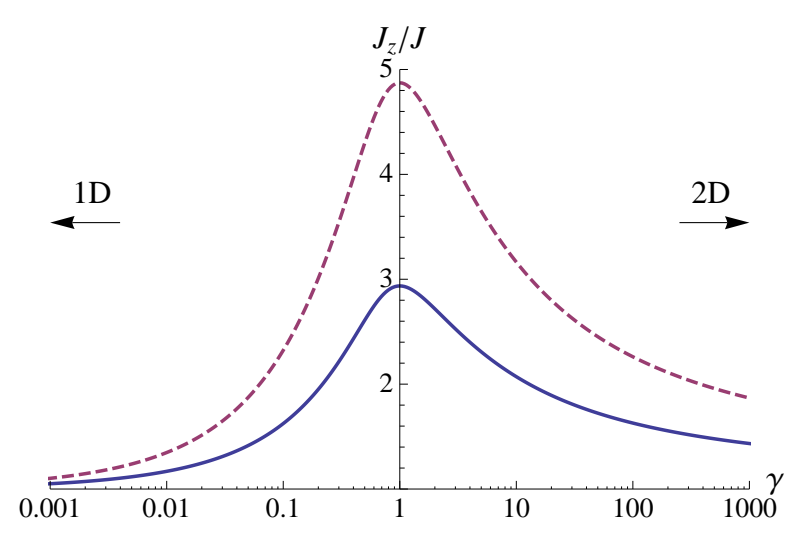

FIG. 4. Critical exchange anisotropy for the twomagnon resonance in the presence of the spatial anisotropy. The solid and dashed curves are for $S=1 / 2$ and $S=1$ with $D=0$, respectively. An $s$-wave bound state at $\boldsymbol{K}=\mathbf{0}$ appears in the upper side of the critical curve.

couplings:

$$
H_{\text {intra }}=-\sum_{\boldsymbol{r}}\left(J^{\hat{z}} \boldsymbol{S}_{\boldsymbol{r}} \cdot \boldsymbol{S}_{\boldsymbol{r}+\hat{\boldsymbol{z}}}-J^{2 \hat{z}} \boldsymbol{S}_{\boldsymbol{r}} \cdot \boldsymbol{S}_{\boldsymbol{r}+2 \hat{\boldsymbol{z}}}\right),
$$

which are realized in $\mathrm{Rb}_{2} \mathrm{Cu}_{2} \mathrm{Mo}_{3} \mathrm{O}_{12}$ [39, $\mathrm{LiCuVO}_{4}$ [40, and $\mathrm{Li}_{2} \mathrm{CuO}_{2}$ [1]. When $J^{\hat{z}} / J^{2 \hat{z}}<4$, the single-magnon dispersion develops minima at nonzero momentum $k_{0} \equiv$ $\pm \arccos \left(J^{\hat{\boldsymbol{z}}} / 4 J^{2 \hat{\boldsymbol{z}}}\right)$, and accordingly, two magnons form a bound state with a center-of-mass momentum $2 k_{0}$ [4147. This two-magnon bound state disappears into the continuum at a certain interchain coupling:

$$
H_{\text {inter }}=-\sum_{\boldsymbol{r}} J\left(\boldsymbol{S}_{\boldsymbol{r}} \cdot \boldsymbol{S}_{\boldsymbol{r}+\hat{\boldsymbol{x}}}+\boldsymbol{S}_{\boldsymbol{r}} \cdot \boldsymbol{S}_{\boldsymbol{r}+\hat{\boldsymbol{y}}}\right),
$$

which leads to the two-magnon resonance. The corresponding critical spatial anisotropy $\gamma=J / J^{\hat{z}}$ is shown in Fig. 5. Because the frustration ratio $J^{\hat{z}} / J^{2 \hat{z}}$ is highly tunable with pressure owing to the strong dependence of ferromagnetic couplings on the cation-anion-cation angle of the superexchange path [48], $S=1 / 2$ frustrated magnets are promising candidates for realizing the Efimov effect without the strong exchange anisotropy. The same approach can also be applied to quasi-two-dimensional frustrated magnets 49 .

So far multi-magnon bound states have been observed mostly in quasi-one-dimensional compounds but with different experimental techniques, such as absorption spectroscopy [6-12, 15, 17, inelastic neutron scattering [18, 21 and electron spin resonance [13, 14, 16, 19, 20, 22, 23. The same spectroscopic measurements can be used here to observe the emergent Efimov states of magnons, provided that the conservation of the magnetization along the magnetic field axis is weakly violated $[8,9,20$. We found that even the lowest bound state of three magnons is already consistent with the universal Efimov state. Its binding energy for ferromagnetic cases is $5 \sim 55 \%$ of the

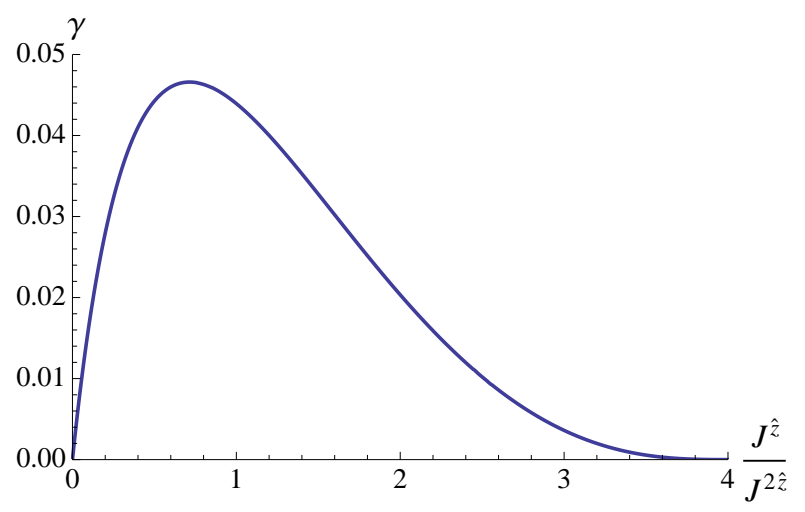

FIG. 5. Critical spatial anisotropy for the two-magnon resonance for $S=1 / 2$ in the presence of the frustration. An $s$-wave bound state at $\boldsymbol{K}=\left(0,0,2 k_{0}\right)$ appears in the lower side of the critical curve. The critical spatial anisotropy for $S=1$ with $D=0$ is at most $\gamma<3.61 \times 10^{-5}$ and thus invisible here.

exchange coupling, which can be up to $10^{2} \sim 10^{3} \mathrm{~K}$. As a dilution refrigerator can lower the temperature down to a few tens of millikelvin, the observation of the lowest one or two Efimov state(s) is within reach.

\section{Methods}

Two-magnon problem

The Schrödinger equation (3) for two magnons $(N=2)$ can be solved in a standard way. By treating the first term in the right hand side as a free part $\left(H_{0}\right)$ and the rest as an interaction part $(V)$, the two-magnon Schrödinger equation can be brought into the Lippmann-Schwinger equation:

$$
\begin{aligned}
& \psi_{\boldsymbol{K}}(\boldsymbol{\rho})=\phi_{\boldsymbol{K}}(\boldsymbol{\rho})+\left\langle\boldsymbol{\rho}\left|\frac{1}{E-H_{0}+i 0^{+}} V\right| \psi_{\boldsymbol{K}}\right\rangle \\
& =\phi_{\boldsymbol{K}}(\boldsymbol{\rho})+\int_{-\pi / a}^{\pi / a} \frac{d \boldsymbol{k}}{(2 \pi / a)^{3}} \frac{\cos (\boldsymbol{k} \cdot \boldsymbol{\rho})}{E-E_{\boldsymbol{K}}(\boldsymbol{k})+i 0^{+}} \\
& \times\left[\sum_{\hat{\boldsymbol{e}}}\left\{J \cos \left(\frac{\boldsymbol{K}}{2} \cdot \hat{\boldsymbol{e}}\right)-J_{z} \cos (\boldsymbol{k} \cdot \hat{\boldsymbol{e}})\right\} \psi_{\boldsymbol{K}}(\hat{\boldsymbol{e}})-2 D \psi_{\boldsymbol{K}}(\mathbf{0})\right] .
\end{aligned}
$$

Here $\phi_{\boldsymbol{K}}(\boldsymbol{\rho})$ is a solution to $E \phi_{\boldsymbol{K}}(\boldsymbol{\rho})=H_{0} \phi_{\boldsymbol{K}}(\boldsymbol{\rho})$ and $E_{\boldsymbol{K}}(\boldsymbol{k}) \equiv E_{0}\left(\frac{\boldsymbol{K}}{2}+\boldsymbol{k}\right)+E_{0}\left(\frac{\boldsymbol{K}}{2}-\boldsymbol{k}\right)$ is the energy of two non-interacting magnons. By setting $\boldsymbol{\rho}=\hat{\boldsymbol{x}}, \hat{\boldsymbol{y}}, \hat{\boldsymbol{z}}$ and $\boldsymbol{\rho}=\mathbf{0}$ in equation (11), we obtain four coupled equations which determine the four unknown constants; $\psi_{\boldsymbol{K}}(\hat{\boldsymbol{e}})$ and $\psi_{\boldsymbol{K}}(\mathbf{0})$, which in turn determine $\psi_{\boldsymbol{K}}(\boldsymbol{\rho})$ through equation (11). The same result was obtained in the isotropic case of $J_{z}=J$ and $D=0$ in the pioneering work [50. 


\section{Three-magnon problem}

The Schrödinger equation (3) for three magnons $(N=$ 3) can be solved in a similar way. By treating the first term in the right hand side as a free part $\left(H_{0}\right)$ and the rest as an interaction part $(V)$, the Lippmann-Schwinger equation for $E<0$ is written as

$$
\Psi\left(\boldsymbol{r}_{1}, \boldsymbol{r}_{2}, \boldsymbol{r}_{3}\right)=\left\langle\boldsymbol{r}_{1}, \boldsymbol{r}_{2}, \boldsymbol{r}_{3}\left|\frac{1}{E-H_{0}} V\right| \Psi\right\rangle .
$$

We then introduce a new parametrization of the wave function:

$$
\begin{aligned}
\chi_{\boldsymbol{K}}(\boldsymbol{\rho} ; \boldsymbol{k}) \equiv & \sum_{\boldsymbol{r}_{1}, \boldsymbol{r}_{2}, \boldsymbol{r}_{3}} \delta_{\boldsymbol{\rho}, \boldsymbol{r}_{1}-\boldsymbol{r}_{2}} e^{-i(\boldsymbol{K}-\boldsymbol{k}) \cdot\left(\frac{\boldsymbol{r}_{1}+\boldsymbol{r}_{2}}{2}\right)-i \boldsymbol{k} \cdot \boldsymbol{r}_{3}} \\
& \times \Psi\left(\boldsymbol{r}_{1}, \boldsymbol{r}_{2}, \boldsymbol{r}_{3}\right)
\end{aligned}
$$

which describes three magnons with a center-of-mass momentum $\boldsymbol{K}$ in which two of them are separated by a distance $\boldsymbol{\rho}$ and the third one has a momentum $\boldsymbol{k}$. The Bose statistics of magnons implies $\chi_{\boldsymbol{K}}(\boldsymbol{\rho} ; \boldsymbol{k})=\chi_{\boldsymbol{K}}(-\boldsymbol{\rho} ; \boldsymbol{k})$.

After a straightforward calculation, the LippmannSchwinger equation (12) can be brought into

$$
\begin{aligned}
& \chi_{\boldsymbol{K}}\left(\boldsymbol{\rho} ; \boldsymbol{k}_{3}\right)=\int_{-\pi / a}^{\pi / a} \frac{d \boldsymbol{k}_{1}}{(2 \pi / a)^{3}} \frac{\cos \left(\frac{2 \boldsymbol{k}_{1}+\boldsymbol{k}_{3}-\boldsymbol{K}}{2} \cdot \boldsymbol{\rho}\right)}{E-E_{0}\left(\boldsymbol{k}_{1}, \boldsymbol{K}-\boldsymbol{k}_{1}-\boldsymbol{k}_{3}, \boldsymbol{k}_{3}\right)} \\
& \times\left[\sum_{\hat{\boldsymbol{e}}}\left\{J \cos \left(\frac{\boldsymbol{K}-\boldsymbol{k}_{3}}{2} \cdot \hat{\boldsymbol{e}}\right)-J_{z} \cos \left(\frac{2 \boldsymbol{k}_{1}+\boldsymbol{k}_{3}-\boldsymbol{K}}{2} \cdot \hat{\boldsymbol{e}}\right)\right\} \chi_{\boldsymbol{K}}\left(\hat{\boldsymbol{e}} ; \boldsymbol{k}_{3}\right)\right. \\
& \quad+2 \sum_{\hat{\boldsymbol{e}}}\left\{J \cos \left(\frac{\boldsymbol{K}-\boldsymbol{k}_{1}}{2} \cdot \hat{\boldsymbol{e}}\right)-J_{z} \cos \left(\frac{\boldsymbol{k}_{1}+2 \boldsymbol{k}_{3}-\boldsymbol{K}}{2} \cdot \hat{\boldsymbol{e}}\right)\right\} \\
& \left.\quad \times \chi_{\boldsymbol{K}}\left(\hat{\boldsymbol{e}} ; \boldsymbol{k}_{1}\right)-2 D \chi_{\boldsymbol{K}}\left(\mathbf{0} ; \boldsymbol{k}_{3}\right)-4 D \chi_{\boldsymbol{K}}\left(\mathbf{0} ; \boldsymbol{k}_{1}\right)\right]
\end{aligned}
$$

where $E_{0}\left(\boldsymbol{k}_{1}, \boldsymbol{k}_{2}, \boldsymbol{k}_{3}\right) \equiv \sum_{i=1,2,3} E_{0}\left(\boldsymbol{k}_{i}\right)$ is the energy of three non-interacting magnons. By setting $\boldsymbol{\rho}=\hat{\boldsymbol{x}}, \hat{\boldsymbol{y}}, \hat{\boldsymbol{z}}$ and $\boldsymbol{\rho}=\mathbf{0}$ in equation (14), we obtain four coupled integral equations which determine the allowed binding energy $E<0$ and the four unknown functions; $\chi_{\boldsymbol{K}}(\hat{\boldsymbol{e}} ; \boldsymbol{k})$ and $\chi_{\boldsymbol{K}}(\mathbf{0} ; \boldsymbol{k})$, which in turn determine $\chi_{\boldsymbol{K}}(\boldsymbol{\rho} ; \boldsymbol{k})$ through equation (14).

Bound-state solutions in the $s$-wave channel at $\boldsymbol{K}=\mathbf{0}$ correspond to those where (i) $\chi_{\mathbf{0}}\left(\hat{\boldsymbol{x}} ; k_{x}, k_{y}, k_{z}\right)=\chi_{\mathbf{0}}\left(\hat{\boldsymbol{y}} ; k_{z}, k_{x}, k_{y}\right)=\chi_{\mathbf{0}}\left(\hat{z} ; k_{y}, k_{z}, k_{x}\right)$, (ii) $\chi_{\mathbf{0}}\left(\hat{\boldsymbol{x}} ; k_{x}, k_{y}, k_{z}\right)=\chi_{\mathbf{0}}\left(\hat{\boldsymbol{x}} ; k_{x}, k_{z}, k_{y}\right)$, (iii) $\chi_{\mathbf{0}}\left(\mathbf{0} ; k_{x}, k_{y}, k_{z}\right)$ is symmetric under any exchange among $\left\{k_{x}, k_{y}, k_{z}\right\}$ and (iv) all of them are even functions of $k_{\nu}(\nu=x, y, z)$. The resulting two coupled integral equations with three variables ranging from 0 to $\pi$ are solved numerically by discretizing each variable with the Gaussian quadrature rule.

\section{Acknowledgements}

This work was supported by a LANL Oppenheimer Fellowship and the US DOE contract No. DE-AC52-
06NA25396 through the LDRD program. All authors contributed equally to this work and declare no competing financial interests. Correspondence and requests for materials should be addressed to Y.N.

* nishida@yukawa.kyoto-u.ac.jp

[1] Fisher, M. E. Renormalization group theory: Its basis and formulation in statistical physics. Rev. Mod. Phys. 70, 653-681 (1998).

[2] Efimov, V. Energy levels arising from resonant two-body forces in a three-body system. Phys. Lett. B 33, 563-564 (1970).

[3] Nielsen, E., Fedorov, D. V., Jensen, A. S. \& Garrido, E. The three-body problem with short-range interactions. Phys. Rept. 347, 373-459 (2001).

[4] Braaten, E. \& Hammer, H-W. Universality in few-body systems with large scattering length. Phys. Rept. 428, 259-390 (2006).

[5] Kraemer, T. et al. Evidence for Efimov quantum states in an ultracold gas of caesium atoms. Nature 440, 315-318 (2006)

[6] Date, M. \& Motokawa, M. Spin-cluster resonance in $\mathrm{CoCl}_{2} \cdot 2 \mathrm{H}_{2} \mathrm{O}$. Phys. Rev. Lett. 16, 1111-1114 (1966).

[7] Date, M. \& Motokawa, M. Spin-cluster resonance in the Ising spin system. J. Phys. Soc. Jpn 24, 41-50 (1968).

[8] Torrance, J. B. Jr \& Tinkham, M. Magnon bound states in anisotropic linear chains. Phys. Rev. 187, 587-594 (1969).

[9] Torrance, J. B. Jr \& Tinkham, M. Excitation of multiplemagnon bound states in $\mathrm{CoCl}_{2} \cdot 2 \mathrm{H}_{2} \mathrm{O}$. Phys. Rev. 187, 595-606 (1969).

[10] Nicoli, D. F. \& Tinkham, M. Far-infrared laser spectroscopy of the linear Ising system $\mathrm{CoCl}_{2} \cdot 2 \mathrm{H}_{2} \mathrm{O}$. Phys. Rev. B 9, 3126-3140 (1974).

[11] Van Vlimmeren, Q. A. G. \& de Jonge, W. J. M. Spincluster resonance in the pseudo-one-dimensional canted Ising antiferromagnet $\mathrm{RbFeCl}_{3} \cdot 2 \mathrm{H}_{2} \mathrm{O}$. Phys. Rev. B 19, 1503-1514 (1979).

[12] Van Vlimmeren, Q. A. G. et al. Spin-cluster excitations in $\mathrm{RbFeCl}_{3} \cdot 2 \mathrm{H}_{2}$ O. Phys. Rev. B 21, 3005-3014 (1980).

[13] Hoogerbeets, R., van Duyneveldt, A. J., Phaff, A. C., Swuste, C. H. W. \& de Jonge, W. J. M. Evidence for magnon bound-state excitations in the quantum chain system $\left(\mathrm{C}_{6} \mathrm{H}_{11} \mathrm{NH}_{3}\right) \mathrm{CuCl}_{3}$. J. Phys. C: Solid State Phys. 17, 2595-2608 (1984).

[14] Nijhof, E. J., van der Vlist, H., Puértolas, J. A. \& Gerritsma, G. J. Magnetism in the linear-chain antiferromagnet $\mathrm{RbCoCl}_{3} \cdot 2 \mathrm{H}_{2} \mathrm{O}$ studied by dynamic susceptibility and electron-spin resonance. Phys. Rev. B 33, 48544867 (1986).

[15] Bosch, L. A., Lauwers, G. J. P. M., Kopinga, K., van der Steen, C. \& de Jonge, W. J. M. The far-infrared magnetic excitation spectrum of $\mathrm{CoCl}_{2}\left(\mathrm{NC}_{5} \mathrm{H}_{5}\right)_{2}$. J. Phys. C: Solid State Phys. 20, 609-627 (1987).

[16] Rubins, R. S., Black, T. D., Sohn, A. \& Drumheller, J. E. Spin-cluster-resonance spectra in an $S=2$ onedimensional Ising ferromagnet. Phys. Rev. B 49, 1536615369 (1994).

[17] Perkins, J. D. et al. Infrared optical excitations in $\mathrm{La}_{2} \mathrm{NiO}_{4}$. Phys. Rev. B 52, R9863-R9866 (1995). 
[18] Garrett, A. W., Nagler, S. E., Tennant, D. A., Sales, B. C. \& Barnes, T. Magnetic excitations in the $S=1 / 2$ alternating chain compound $(\mathrm{VO})_{2} \mathrm{P}_{2} \mathrm{O}_{7}$. Phys. Rev. Lett. 79, 745-748 (1997).

[19] Orendáč, M. et al. Single-ion bound states in $S=$ 1 Heisenberg antiferromagnetic chains with planar anisotropy and subcritical exchange coupling. Phys. Rev. $B$ 60, 4170-4175 (1999).

[20] Katsumata, K. et al. Single-ion magnon bound states in an antiferromagnet with strong uniaxial anisotropy. Phys. Rev. B 61, 11632-11636 (2000).

[21] Tennant, D. A. et al. Neutron scattering study of twomagnon states in the quantum magnet copper nitrate. Phys. Rev. B 67, 054414 (2003).

[22] Zvyagin, S. A. et al. Magnetic excitations in the spin1 anisotropic Heisenberg antiferromagnetic chain system $\mathrm{NiCl}_{2}-4 \mathrm{SC}\left(\mathrm{NH}_{2}\right)_{2}$. Phys. Rev. Lett. 98, 047205 (2007).

[23] Psaroudaki, C. et al. Magnetic excitations in the spin1 anisotropic antiferromagnet $\mathrm{NiCl}_{2}-4 \mathrm{SC}\left(\mathrm{NH}_{2}\right)_{2}$. Phys. Rev. B 85, 014412 (2012).

[24] Giamarchi, T., Rüegg, C. \& Tchernyshyov, O. BoseEinstein condensation in magnetic insulators. Nature Phys. 4, 198-204 (2008).

[25] Holstein, T. \& Primakoff, H. Field dependence of the intrinsic domain magnetization of a ferromagnet. Phys. Rev. 58, 1098-1113 (1940).

[26] Watson, G. N. Three triple integrals. Q. J. Math. 10, 266-276 (1939).

[27] Hammer, H-W. \& Platter, L. Universal properties of the four-body system with large scattering length. Eur. Phys. J. A 32, 113-120 (2007).

[28] Von Stecher, J., D'Incao, J. P. \& Greene, C. H. Signatures of universal four-body phenomena and their relation to the Efimov effect. Nature Phys. 5, 417-421 (2009).

[29] Blundell, S. J. \& Pratt, F. L. Organic and molecular magnets. J. Phys.: Condens. Matter 16, R771-R828 (2004).

[30] Koch, R., Waldmann, O., Müller, P., Reimann, U. \& Saalfrank, R. W. Ferromagnetic coupling and magnetic anisotropy in molecular Ni(II) squares. Phys. Rev. B 67, 094407 (2003).

[31] Kawamoto, T., Tokumoto, M., Sakamoto, H. \& Mizoguchi, K. Theoretical study of pressure effect on TDAE-C 60 . J. Phys. Soc. Jpn 70, 1892-1895 (2001).

[32] Landee, C. P. \& Willett, R. D. Tetramethylammonium copper chloride and tris (trimethylammonium) copper chloride: $S=1 / 2$ Heisenberg one-dimensional ferromagnets. Phys. Rev. Lett. 43, 463-466 (1979).

[33] Takahashi, M. et al. Discovery of a quasi-1D organic ferromagnet, $p$-NPNN. Phys. Rev. Lett. 67, 746-748 (1991).

[34] Feldkemper, S., Weber, W., Schulenburg, J. \& Richter, J. Ferromagnetic coupling in nonmetallic $\mathrm{Cu}^{2+}$ compounds. Phys. Rev. B 52, 313-323 (1995).
[35] Manaka, H., Koide, T., Shidara, T. \& Yamada, I. Observation of polarization-dependent x-ray absorption spectra arising from $\mathrm{Cu} 3 d-\mathrm{F} 2 p$ hybridization in the two-dimensional ferromagnets $\mathrm{A}_{2} \mathrm{CuF}_{4}(\mathrm{~A}=\mathrm{K}, \mathrm{Cs})$. Phys. Rev. B 68, 184412 (2003).

[36] Shimizu, K. et al. Ferromagnetic ordering of $S=1 / 2$ Heisenberg ferromagnetic chains in organic magnet $\beta$ BBDTA.GaBr 4 . Phys. Rev. B 74, 172413 (2006).

[37] Sugano, T., Blundell, S. J., Lancaster, T., Pratt, F. L. \& Mori, H. Magnetic order in the purely organic quasione-dimensional ferromagnet 2-benzimidazolyl nitronyl nitroxide. Phys. Rev. B 82, 180401(R) (2010).

[38] Pradeep, T. Advances in Physical Chemistry (Allied Publishers, New Delhi, 1999).

[39] Hase, M. et al. Magnetic properties of $\mathrm{Rb}_{2} \mathrm{Cu}_{2} \mathrm{Mo}_{3} \mathrm{O}_{12}$ including a one-dimensional spin-1/2 Heisenberg system with ferromagnetic first-nearest-neighbor and antiferromagnetic second-nearest-neighbor exchange interactions. Phys. Rev. B 70, 104426 (2004).

[40] Enderle, M. et al. Quantum helimagnetism of the frustrated spin-1/2 chain $\mathrm{LiCuVO}_{4}$. Europhys. Lett. 70, 237243 (2005).

[41] Dmitriev, D. V. \& Krivnov, V. Ya. Multimagnon bound states in an easy-axis frustrated ferromagnetic spin chain. Phys. Rev. B 79, 054421 (2009).

[42] Bahurmuz, A. A. \& Loly, P. D. The complete two-magnon spectrum of the ferromagnetic Heisenberg chain including NNN interactions. J. Phys. C: Solid State Phys. 19, 2241-2252 (1986).

[43] Chubukov, A. V. Chiral, nematic, and dimer states in quantum spin chains. Phys. Rev. B 44, 4693-4696 (1991).

[44] Cabra, D. C., Honecker, A. \& Pujol, P. Magnetic properties of zig-zag ladders. Eur. Phys. J. B 13, 55-73 (2000).

[45] Dmitriev, D. V. \& Krivnov, V. Ya. Frustrated ferromagnetic spin-1/2 chain in a magnetic field. Phys. Rev. B 73, 024402 (2006).

[46] Kuzian, R. O. \& Drechsler, S-L. Exact one- and twoparticle excitation spectra of acute-angle helimagnets above their saturation magnetic field. Phys. Rev. B 75, 024401 (2007).

[47] Kecke, L., Momoi, T. \& Furusaki, A. Multimagnon bound states in the frustrated ferromagnetic onedimensional chain. Phys. Rev. B 76, 060407(R) (2007).

[48] Goodenough, J. B. Magnetism and the Chemical Bond (John Wiley \& Sons, New York, 1963).

[49] Nath, R., Tsirlin, A. A., Rosner, H. \& Geibel, C. Magnetic properties of $\mathrm{BaCdVO}\left(\mathrm{PO}_{4}\right)_{2}$ : A strongly frustrated spin- $1 / 2$ square lattice close to the quantum critical regime. Phys. Rev. B 78, 064422 (2008).

[50] Wortis, M. Bound states of two spin waves in the Heisenberg ferromagnet. Phys. Rev. 132, 85-97 (1963). 